\section{(2) OPEN ACCESS}

\title{
Effectiveness and safety of oral anticoagulants in elderly patients with atrial fibrillation
}

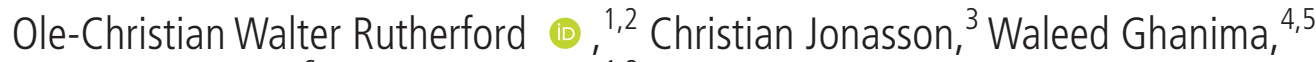 \\ Fabian Söderdahl, ${ }^{6}$ Sigrun Halvorsen ${ }^{1,2}$
}

\begin{abstract}
- Additional supplemental material is published online only. To view, please visit the journal online (http://dx.doi. org/10.1136/heartjnl-2020318753).
\end{abstract}

${ }^{1}$ Department of Cardiology, Oslo University Hospital Ulleval, Oslo, Norway

${ }^{2}$ Institute of Clinical Medicine, University of Oslo, Oslo, Norway ${ }^{3}$ HUNT Research Center, Faculty of Medicine, NTNU - Norwegian University of Science and

Technology, Trondheim, Norway ${ }^{4}$ Department of Research, Internal Medicine, Ostfold Hospital Trust, Sarpsborg, Norway

${ }^{5}$ Department of Haematology, Institute of Clinical Medicine University of Oslo, Oslo, Norway ${ }^{6}$ Statisticon AB, Stockholm, Sweden

\section{Correspondence to}

Dr Ole-Christian Walter Rutherford, Cardiology, Oslo University Hospital Ulleval, Oslo, Oslo, Norway;

ole-christian.rutherford@so-hf. no

Received 2 December 2020 Revised 4 April 2021

Accepted 16 April 2021

\section{ABSTRACT \\ Objectives To assess the risk of stroke/systemic} embolism (SE) and major bleeding associated with the use of oral anticoagulants in elderly patients with atrial fibrillation (AF) in a real-world population.

Methods We identified all anticoagulant-naive initiators of warfarin, dabigatran, rivaroxaban and apixaban for the indication AF in Norway between January 2013 and December 2017. Multivariate competing risk regression was used to calculate subhazard ratios (SHRs) describing associations between non-vitamin $\mathrm{K}$ antagonist oral anticoagulants (NOACs) compared with warfarin for risk of stroke/SE and major bleeding.

Results Among 30401 patients $\geq 75$ years identified (median age 82 years, 53\% women, mean $\mathrm{CHA}_{2} \mathrm{DS}_{2}$-VaSc score 4.5), 3857 initiated dabigatran, 6108 rivaroxaban, 13786 apixaban and 6650 warfarin. Reduced dose was initiated in 11559 (49\%) of the NOAC-treated patients. For stroke, the SHRs for standard dose NOAC against warfarin were $0.80(95 \% \mathrm{Cl} 0.57$ to 1.13$)$ for dabigatran; 1.07 (95\% Cl 0.89 to 1.30 ) for rivaroxaban and 0.95 (95\% Cl 0.78 to 1.15$)$ for apixaban. For major bleeding, the SHRs against warfarin were 0.75 (95\% $\mathrm{Cl} 0.52$ to 1.08$)$ for dabigatran; $0.96(95 \% \mathrm{Cl} 0.78$ to 1.16) for rivaroxaban and $0.74(95 \% \mathrm{Cl} 0.60$ to 0.91$)$ for apixaban. Comparing reduced doses of NOACs with warfarin yielded similar results. Sensitivity analyses were in accordance with the main results.

Conclusion In this nationwide cohort study of patients $\geq 75$ years initiating oral anticoagulation for $A F$, standard and reduced dose NOACs were associated with similar risks of stroke/SE as warfarin and lower or similar risks of bleeding. The NOACs seem to be a safe option also in elderly patients.

\section{INTRODUCTION}

Age is a strong and independent risk factor for both stroke and bleeding in patients with atrial fibrillation (AF). ${ }^{1}$ Oral anticoagulation is associated with a net clinical benefit in elderly patients despite their elevated bleeding risk, ${ }^{2}$ and the 2020 European Society of Cardiology (ESC) guidelines for the management of AF recommend non-vitamin $\mathrm{K}$ antagonist oral anticoagulants (NOACs) for stroke prevention over vitamin $\mathrm{K}$ antagonists (VKAs), without age restrictions. ${ }^{3}$

In the pivotal randomised controlled trials (RCTs) leading to the approval of the NOACs, the median age was just over 70 years and approximately $65 \%$ of the patients included were men. ${ }^{4-6}$
In the real world, approximately half of patients with AF starting on oral anticoagulants (OACs) are 75 years or older, and approximately half of these are women. ${ }^{7}$ No RCT has investigated the efficacy and safety of NOACs specifically in elderly patients, but subgroup analyses of the RCTs, ${ }^{8-11}$ and observational studies, ${ }^{12} 13$ indicate that the benefits of NOACs over VKAs are maintained in the elderly population. More insight into the comparative abilities of anticoagulants to reduce the risk of stroke while keeping bleeding risk low in elderly patients is needed.

In this study, we aimed to compare the risks of stroke or systemic embolism (SE), and major bleeding, between standard and reduced doses of dabigatran, rivaroxaban, apixaban and warfarin, in a Norwegian nationwide cohort of patients $\geq 75$ years with AF. In Norway, data from all hospital contacts and prescription dispensations are routinely collected through national registries, ${ }^{14}$ making it possible to follow individuals over time with virtually no selection bias.

\section{METHODS}

\section{Data sources}

Data were collected from the Norwegian Patient Registry (NPR) and the Norwegian Prescription Database (NorPD). The NPR contains diagnoses from all hospital admissions, outpatient consultations and specialist consultations in Norway. ${ }^{14}$ For each contact, the primary (the primary disease/ condition treated) and secondary codes (relevant comorbidities) are recorded. Diagnoses are coded according to the International Classification of Diseases (10th revision, ICD-10) system, and surgical procedures according to the Nordic Medico-Statistical Committee (NOMESCO) coding system. $^{15}$

The NorPD contains information from all pharmacies in Norway on dispensations including drug codes (Anatomical Therapeutic Chemical system (ATC)), drug strength, pack-size and vital status of patients. ${ }^{16}$ Drug expenses for treatment of serious chronic illnesses are reimbursed in Norway, and the NorPD contains the relevant ICD-10/International Classification of Primary Care (ICPC-2) codes warranting reimbursement. Linkage of individuallevel data across NPR and NorPD was enabled via unique personal identification numbers.

\section{Cohort creation and study design}

All patients diagnosed with AF, but without mitral stenosis or mechanical heart valves, between January 


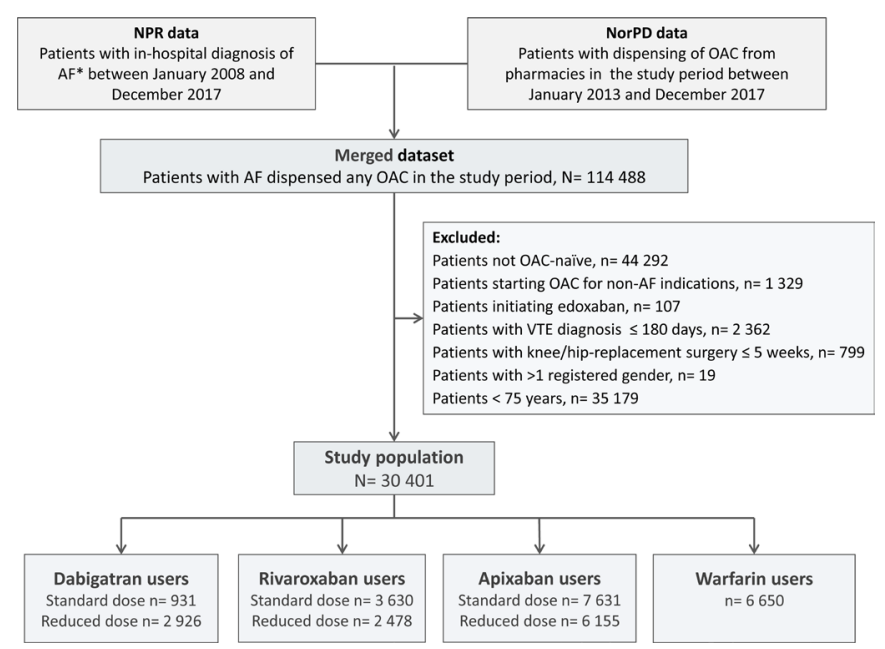

Figure 1 Cohort creation flow-chart. AF*, atrial fibrillation in the absence of mitral stenosis or mechanical prosthetic heart valves; NPR, Norwegian Patient Registry; NorPD, Norwegian Prescription Database; OAC, oral anticoagulant; VTE, venous thromboembolism.

2008 and December 2017 were identified from the NPR. From the NorPD, we identified all patients with at least one dispensation of an OAC between January 2013 and December 2017. These data were linked to create a cohort of patients diagnosed with $\mathrm{AF}$, initiating treatment with an OAC (figure 1). The index date was set to the day of the first dispensing of an OAC (dabigatran $110 \mathrm{mg} / 150 \mathrm{mg}$, rivaroxaban $15 \mathrm{mg} / 20 \mathrm{mg}$, apixaban 2.5 $\mathrm{mg} / 5 \mathrm{mg}$ or warfarin $2.5 \mathrm{mg}$ ) for the indication AF in the study period. We chose an 'active-comparator, new-user' design: the drug of interest was compared with another agent used for the same indication rather than with no treatment. This ensures that treatment groups have similar treatment indications, minimising differences in patient characteristics. With the new-user design, patients were included from the time of treatment initiation, enabling capture of all events occurring during follow-up. ${ }^{17}$ The design involves a washout period before inclusion; patients with a dispensing of any anticoagulant in the preceding 12 months before the index date; a history of venous thromboembolism during the last 180 days; or knee or hip replacement surgery during the last 35 days before the index date were excluded. Due to limited usage in the study period, patients initiating edoxaban were excluded $(n=107)$. Finally, all patients $<75$ years were excluded, creating a cohort of anticoagulant-naive AF patients $\geq 75$ years starting treatment with warfarin, dabigatran, rivaroxaban or apixaban (figure 1). ICD-10 codes used for inclusion/exclusion criteria, comorbidities and outcomes are listed in online supplemental table 1 .

\section{OAC supply}

The days of warfarin supply were estimated as previously described. ${ }^{18}$ The period of supply of NOACs was estimated by the pack-size/number of packs prescribed, given a fixed dosing of NOACs. To account for incomplete adherence, a 30-day grace period between the calculated end of NOAC supply and the date of a new prescription was allowed.

\section{Comorbidities}

Using ICD-10-diagnoses from NPR, and ICD/ICPC-2-diagnoses from NorPD, a set of comorbidities was compiled for the last 5 years before the index date for each patient. Online supplemental table S2 shows in detail how $\mathrm{CHA}_{2} \mathrm{DS}_{2}$-VASc and HAS-BLED scores were calculated. For identification of comorbidities, both primary and secondary ICD-10 and NOMESCO codes from the NPR were used.

\section{Outcomes and follow-up period}

The main outcomes investigated were stroke or SE (effectiveness outcome) and major bleeding (safety outcome). Other outcomes included ischaemic stroke, intracranial haemorrhage, gastrointestinal haemorrhage, any haemorrhage and all-cause mortality. Major bleeding was defined as any bleeding into a critical area or organ such as intracranial, intraspinal, intraocular, retroperitoneal, intra-articular, pericardial, intramuscular with compartment syndrome, major gastrointestinal and/or any bleeding accompanied by blood transfusion $\leq 10$ days after hospital admission. For identification of outcomes, only primary (first listed) ICD-10 and NOMESCO codes for each hospital stay were used (online supplemental table S1). Patients were followed until discontinuation or switching of OAC, death or end of study period (31 December 2017), whichever occurred first.

\section{Statistical analysis}

Categorical variables are reported as numbers and per cent, continuous variables as means with SD or medians with 25 th -75 th percentiles. Based on clinical experience and by using directed acyclic graphs, we identified a group of 20 confounders for the effect of exposure to OACs on both the chosen outcomes and the competing risk of death. ${ }^{19}$ Multivariate competing risk regression adjusting for these 20 variables was performed according to the method of Fine and Gray, ${ }^{20}$ to calculate subhazard ratios (SHR) describing associations between exposure to different OACs and the defined outcomes, treating death as a competing risk. The results were graphically presented by cumulative incidence functions. $^{21}$ To evaluate associations between OAC therapy and risk of all-cause mortality, multivariate Cox regression was performed. The proportional hazards assumption was checked using Schoenfeld residuals and by comparing the log-log transformation of the Kaplan-Meier survival curves for each variable. Robust sandwich estimates were calculated. ${ }^{22}$ Estimating days of supply for warfarin, as well as anticoagulant effect of the dose taken, is difficult in registry-based studies. To elaborate on our findings, we performed post hoc analyses with NOAC-NOAC comparisons after the main analyses, which compared NOACs with warfarin. The variables adjusted for were gender, age, year of inclusion into the study, chronic kidney disease, hypertension, diabetes, ischaemic heart disease, peripheral artery disease, heart failure, dementia, thyroid disorders, active cancer (cancer diagnosis last 12 months), chronic lower respiratory tract disease, history of stroke/SE, history of bleeding-related hospitalisation, history of anaemia, use of cholesterol lowering drugs, use of antiplatelet drugs and use of NSAIDs during the last 12 months. Level of significance was set to 5\%. Statistical analyses were performed using SAS V.9.4 (SAS Institute) and STATA V.16 (STATACorp LLC).

\section{Sensitivity analyses}

Four sensitivity analyses were performed for the outcomes stroke/SE, major bleeding and all-cause mortality: (1) allowing a longer gap period of 90 days between the calculated end of OAC supply and a new prescription dispensing before censoring; (2) analysing only truly OAC naive patients, by excluding patients with a dispensing of any anticoagulant for any indication from pharmacies during the last 5 years (12 months was used in the main analyses); (3) standardising follow-up time for all OACs to 
Table 1 Baseline characteristics

\begin{tabular}{|c|c|c|c|c|c|c|c|}
\hline & \multirow{2}{*}{$\begin{array}{l}\text { Dabigatran } \\
150 \mathrm{mg} \text { two times } \\
\text { per day }\end{array}$} & \multirow{2}{*}{$\begin{array}{l}\text { Dabigatran } \\
110 \text { two times } \\
\text { per day }\end{array}$} & \multirow{2}{*}{$\begin{array}{l}\text { Rivaroxaban } \\
20 \mathrm{mg} \text { once } \\
\text { a day }\end{array}$} & \multirow{2}{*}{$\begin{array}{l}\text { Rivaroxaban } \\
15 \mathrm{mg} \text { once } \\
\text { a day }\end{array}$} & \multirow{2}{*}{$\begin{array}{l}\text { Apixaban } \\
5 \mathrm{mg} \text { two times } \\
\text { per day }\end{array}$} & \multirow{2}{*}{$\begin{array}{l}\text { Apixaban } \\
2.5 \mathrm{mg} \text { two times } \\
\text { per day }\end{array}$} & \multirow{2}{*}{$\begin{array}{l}\text { Warfarin } \\
2.5 \mathrm{mg}\end{array}$} \\
\hline & & & & & & & \\
\hline $\mathrm{N}$ & 931 & 2926 & 3630 & 2478 & 7631 & 6155 & 6650 \\
\hline \multicolumn{8}{|l|}{ Year of inclusion into study } \\
\hline 2013 & $356(38.2)$ & $1333(45.6)$ & $902(24.8)$ & $724(29.2)$ & $93(1.2)$ & $87(1.4)$ & $3131(47.1)$ \\
\hline 2014 & $284(30.5)$ & 837 (28.6) & $834(23.0)$ & $653(26.4)$ & $846(11.1)$ & $846(13.7)$ & $1868(28.1)$ \\
\hline 2015 & $116(12.5)$ & $296(10.1)$ & $755(20.8)$ & $539(21.8)$ & $1780(23.3)$ & $1546(25.1)$ & $951(14.3)$ \\
\hline 2016 & $88(9.5)$ & $224(7.7)$ & $680(18.7)$ & $362(14.6)$ & $2303(30.2)$ & $1916(31.1)$ & $485(7.3)$ \\
\hline 2017 & $87(9.3)$ & $236(8.1)$ & $459(12.6)$ & $200(8.1)$ & 2609 (34.2) & $1760(28.6)$ & $215(3.2)$ \\
\hline \multicolumn{8}{|l|}{ Age } \\
\hline $75-84$ & $875(94.0)$ & $1867(63.8)$ & $2764(76.1)$ & 1279 (51.6) & $6020(78.9)$ & $2643(42.9)$ & $4186(62.9)$ \\
\hline $85-94$ & $53(5.7)$ & $1020(34.9)$ & $845(23.3)$ & $1122(45.3)$ & $1560(20.4)$ & $3254(52.9)$ & $2379(35.8)$ \\
\hline $95-105$ & $3(0.3)$ & $39(1.3)$ & $21(0.6)$ & $77(3.1)$ & $51(0.7)$ & $258(4.2)$ & $85(1.3)$ \\
\hline Mean (SD) & $78.0(3.5)$ & $83.0(4.9)$ & $81.0(4.8)$ & $84.4(5.4)$ & $80.8(4.6)$ & $85.6(5.3)$ & $82.9(5.1)$ \\
\hline Median (25th-75th percentile) & 77 (76-79) & $82(79-86)$ & $80(77-84)$ & $84(80-88)$ & $80(77-84)$ & $86(82-89)$ & $82(79-87)$ \\
\hline Female gender & $386(41.5)$ & $1633(55.8)$ & 1812 (49.9) & $1401(56.5)$ & $3744(49.1)$ & $3818(62.0)$ & $3316(49.9)$ \\
\hline Hypertension & $619(66.5)$ & $2194(75.0)$ & $2566(70.7)$ & $1971(79.5)$ & $5521(72.3)$ & $4741(77.0)$ & $5235(78.7)$ \\
\hline Ischaemic heart disease & $186(20.0)$ & $764(26.1)$ & $831(22.9)$ & $750(30.3)$ & $1874(24.6)$ & $1920(31.2)$ & $2511(37.8)$ \\
\hline Peripheral artery disease & $73(7.8)$ & $289(9.9)$ & $366(10.1)$ & $267(10.8)$ & $796(10.4)$ & $722(11.7)$ & $898(13.5)$ \\
\hline Heart failure & $160(17.2)$ & $902(30.8)$ & $830(22.9)$ & $982(39.6)$ & $1979(25.9)$ & $2490(40.5)$ & $2904(43.7)$ \\
\hline Chronic kidney disease & $20(2.1)$ & $146(5.0)$ & $119(3.3)$ & $311(12.6)$ & $387(5.1)$ & $1065(17.3)$ & $1096(16.5)$ \\
\hline Diabetes mellitus & $114(12.2)$ & $377(12.9)$ & $485(13.4)$ & $348(14.0)$ & 1117 (14.6) & $962(15.6)$ & $1187(17.8)$ \\
\hline Thyroid disorders & $32(3.4)$ & $151(5.2)$ & $146(4.0)$ & $143(5.8)$ & $321(4.2)$ & $333(5.4)$ & $385(5.8)$ \\
\hline Chronic lower & $234(25.1)$ & $707(24.2)$ & $944(26.0)$ & $600(24.2)$ & $2081(27.3)$ & 1639 (26.6) & $1760(26.5)$ \\
\hline \multicolumn{8}{|l|}{ respiratory tract disorder } \\
\hline Active cancer (diagnosis last 12 months & $91(9.8)$ & $283(9.7)$ & $375(10.3)$ & $277(11.2)$ & $823(10.8)$ & $715(11.6)$ & $745(11.2)$ \\
\hline Dementia & $12(1.3)$ & $88(3.0)$ & $92(2.5)$ & $94(3.8)$ & $187(2.5)$ & $276(4.5)$ & $203(3.1)$ \\
\hline History of stroke/SE & $136(14.6)$ & $528(18.0)$ & $600(16.5)$ & $411(16.6)$ & $1253(16.4)$ & $1117(18.1)$ & $1096(16.5)$ \\
\hline History of ischaemic stroke & $134(14.4)$ & $512(17.5)$ & $588(16.2)$ & $394(15.9)$ & $1229(16.1)$ & $1087(17.7)$ & $1069(16.1)$ \\
\hline History of intracranial haemorrhage & $3(0.3)$ & $27(0.9)$ & $21(0.6)$ & $23(0.9)$ & $50(0.7)$ & $67(1.1)$ & $45(0.7)$ \\
\hline History of bleeding & $105(11.3)$ & $448(15.3)$ & $501(13.8)$ & $432(17.4)$ & $1106(14.5)$ & $1273(20.7)$ & $1225(18.4)$ \\
\hline History of gastrointestinal bleeding & $23(2.5)$ & $131(4.5)$ & $143(3.9)$ & $143(5.8)$ & $297(3.9)$ & $372(6.0)$ & $399(6.0)$ \\
\hline History of anaemia & $41(4.4)$ & $237(8.1)$ & $236(6.5)$ & $276(11.1)$ & $614(8.0)$ & $812(14.8)$ & $788(11.8)$ \\
\hline Use of antiplatelet drugs last 12 months & $494(53.1)$ & $1789(61.1)$ & $2127(58.6)$ & $1581(63.8)$ & $4384(57.4)$ & $3830(62.2)$ & $3803(57.2)$ \\
\hline Use of NSAIDs last 12 months & $242(26.0)$ & $590(20.2)$ & $770(21.2)$ & $451(18.2)$ & $1584(20.8)$ & $987(16.0)$ & $1128(17.0)$ \\
\hline Use of cholesterol lowering drugs & $461(49.5)$ & $1463(50.0)$ & $1770(48.8)$ & $1264(51.0)$ & $4107(53.8)$ & $3174(51.6)$ & $3735(56.2)$ \\
\hline Mean CHA2DS2-VaSc score (SD) & $3.9(1.3)$ & $4.4(1.4)$ & $4.2(1.3)$ & $4.6(1.4)$ & $4.3(1.3)$ & $4.7(1.4)$ & $4.7(1.4)$ \\
\hline Mean HAS-BLED score (SD) & $2.6(0.95)$ & $2.8(0.9)$ & $2.7(0.95)$ & $3.0(1.0)$ & $2.8(0.98)$ & $3.0(1.1)$ & $2.9(1.0)$ \\
\hline
\end{tabular}

Values are numbers (per cent), unless otherwise stated.

CHA2DS2-VaSc, congestive heart failure (or left ventricular systolic dysfunction), hypertension, age $\geq 75$ years, diabetes mellitus, prior stroke or transient ischaemic attack or systemic embolism, vascular disease, age $\geq 65$ years, sex category; HAS-BLED, hypertension, abnormal renal function/ abnormal liver function, prior stroke, prior major bleeding, labile international normalised ratio (INR), elderly age $\geq 65$ years, prior alcohol or drug abuse/use of medications that predispose to bleeding (antiplatelet agents, NSAIDs); NSAIDs, non-steroidal anti-inflammatory drugs; SE, systemic embolism.

12 months; (4) an 'intention-to-treat'-like analysis, not censoring patients on switching between anticoagulants or discontinuation of therapy.

\section{RESULTS}

In total, 30401 patients were included; 3857 patients initiating dabigatran (standard dose 931 patients; reduced dose 2926); 6108 patients initiating rivaroxaban (standard dose 3630 patients; reduced dose 2478 patients); 13786 patients initiating apixaban (standard dose 7631; reduced dose 6155) and 6650 patients initiating warfarin. The median age for the total population was 82 years (IQR 78-86); the majority of patients were female $(53.0 \%)$, and the mean $\mathrm{CHA}_{2} \mathrm{DS}_{2}$-VASC score was 4.5 (SD 1.4). Baseline characteristics of the study population in relation to treatment groups are shown in table 1. Initiators of standard doses of NOACs were likely to be younger than initiators of warfarin, while initiators of reduced doses of NOACs were more likely to be of similar (dabigatran) or older age (rivaroxaban and apixaban) than initiators of warfarin. Users of dabigatran 150 mg two times per day had the lowest, and users of apixaban $2.5 \mathrm{mg}$ two times per day, the highest median age (77 and 86 years, respectively). Median follow-up time was 24.4 months (standard dose) and 17.8 months (reduced dose) for dabigatran, 19.0 months (standard dose) and 16.2 months (reduced dose) for rivaroxaban, 12.7 months (standard dose) and 11.6 months (reduced dose) for apixaban and 19.9 months for warfarin. The proportion of patients who switched anticoagulants during the study period was 20.3\% (standard dose) and 21.6\% (reduced dose) for dabigatran, $11.8 \%$ (standard dose) and $11.9 \%$ (reduced dose) for rivaroxaban, 2.8\% (standard dose) and 2.7\% (reduced dose) for apixaban and $17.0 \%$ for warfarin. The crude incidence rate of stroke/SE (events per 100 person years) was 


\section{A. Standard dose NOACs}
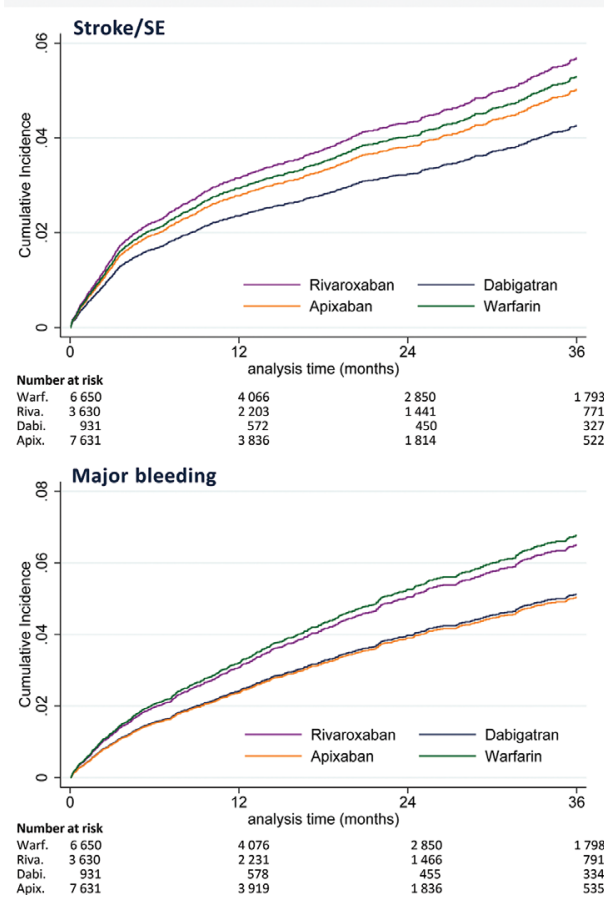

B. Reduced dose NOACs
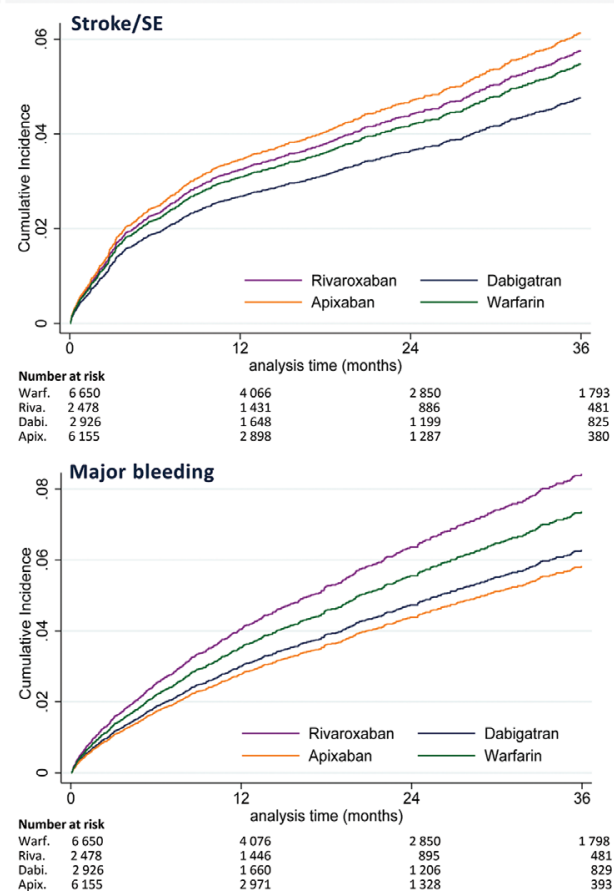

Figure 2 Cumulative incidence of main effectiveness and safety outcomes for warfarin, standard (A) and reduced (B) dose NOACs. NOAC, nonvitamin $\mathrm{K}$ antagonist oral anticoagulant; SE, systemic embolism.

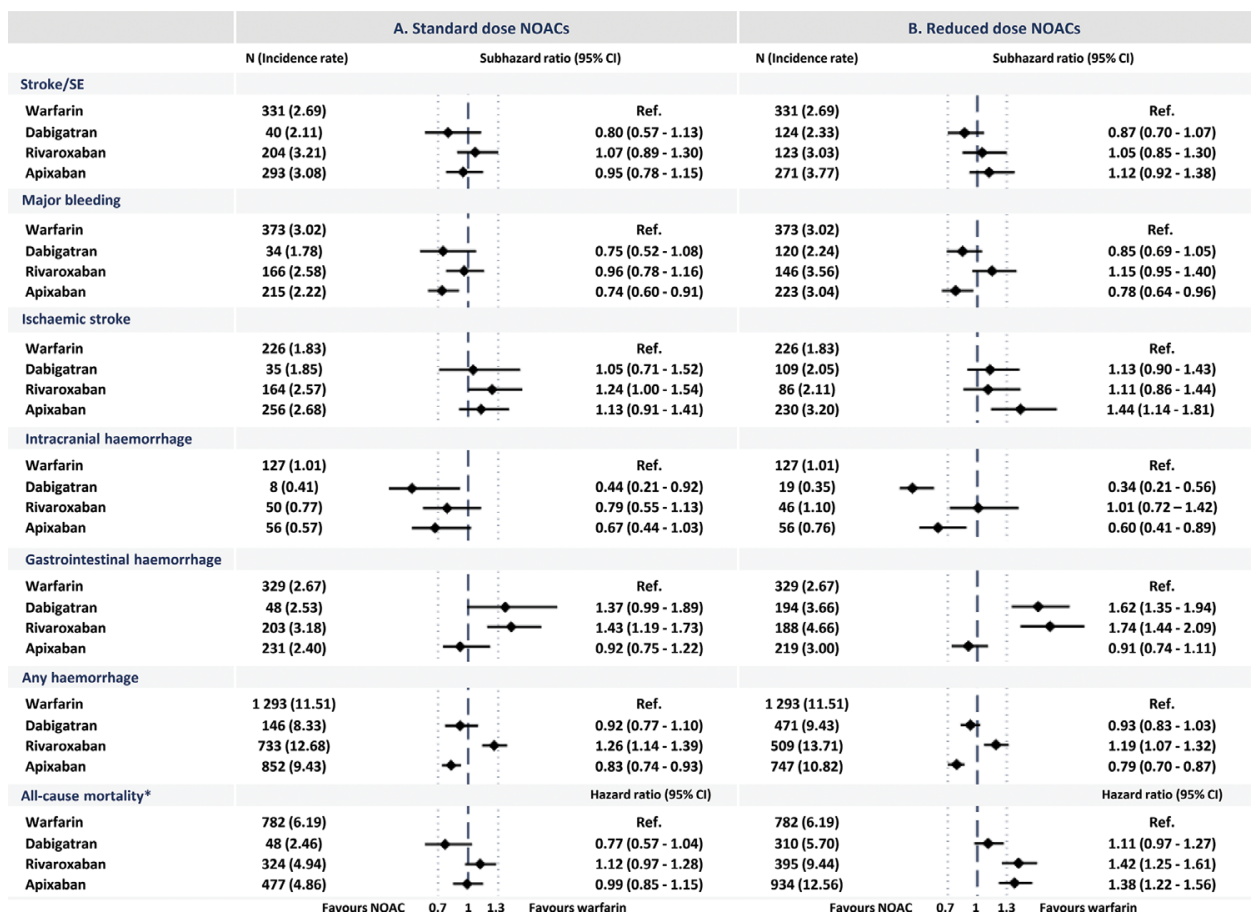

Figure 3 Number of events, crude incidence rates ${ }^{1}$ and subhazard ratios ${ }^{2}$ between standard $(A)$ and reduced (B) dose NOACs and warfarin for all outcomes. ${ }^{1}$ Crude incidence rate, crude incidence/100 patient years; ${ }^{2}$ competing risk regression, treating death as competing risk, adjusted for NOAC dose, gender, age, year of inclusion into the study, chronic kidney disease, hypertension, diabetes, ischaemic heart disease, peripheral artery disease, heart failure, dementia, thyroid disorders, active cancer (cancer diagnosis last 12 months), chronic lower respiratory tract disease, history of stroke/ SE, history of bleeding-related hospitalisation, history of anaemia, use of cholesterol lowering drugs, use of antiplatelet drugs and use of NSAIDs during the last 12 months. *For risk of all-cause mortality multivariate Cox proportional regression adjusting for the same variables used in competing risk regression was performed. NOAC, non-vitamin K antagonist oral anticoagulant; NSAIDs, non-steroidal anti-inflammatory drugs; SE, systemic embolism. 

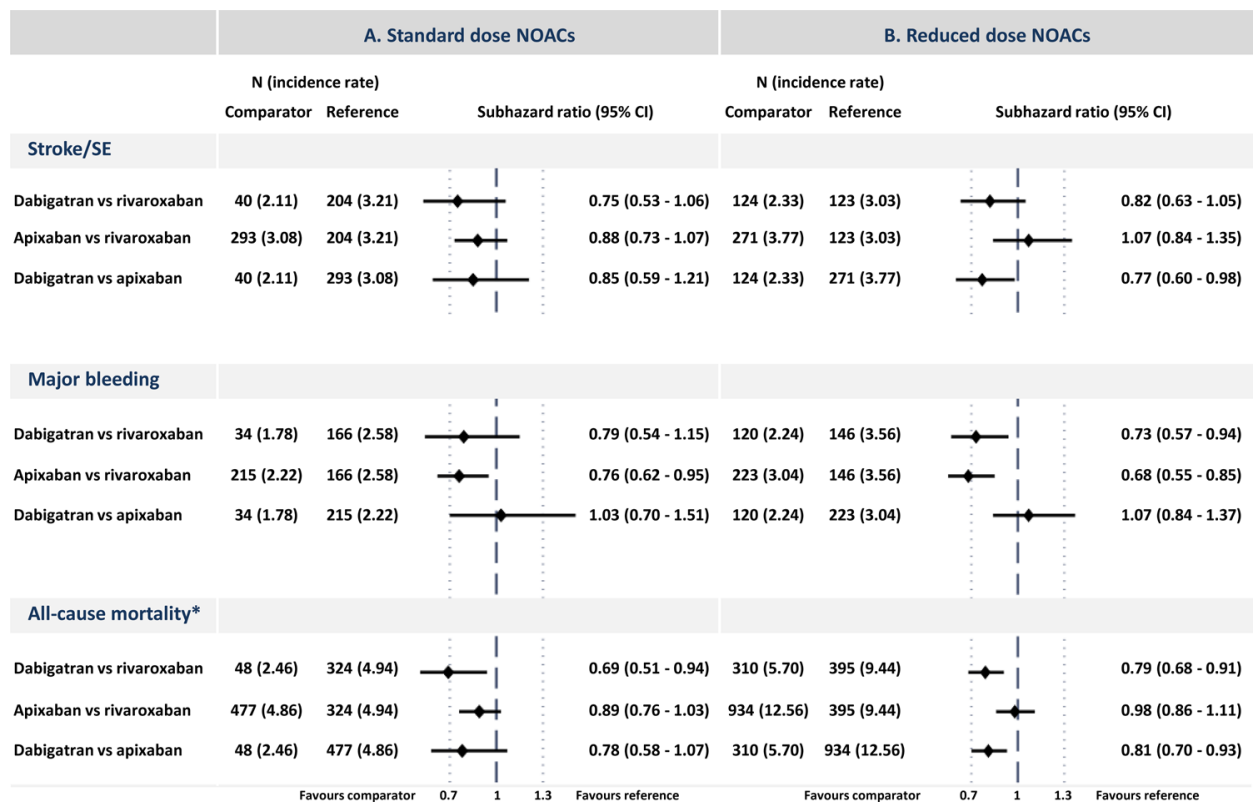

Figure 4 Number of events, crude incidence rates ${ }^{1}$ and subhazard ratios ${ }^{2}$ between standard (A) and reduced (B) dose NOACs for main outcomes and all-cause mortality. ${ }^{1}$ Crude incidence rate, crude incidence/100 patient years; ${ }^{2}$ competing risk regression, treating death as competing risk, adjusted for NOAC dose, gender, age, year of inclusion into the study, chronic kidney disease, hypertension, diabetes, ischaemic heart disease, peripheral artery disease, heart failure, dementia, thyroid disorders, active cancer (cancer diagnosis last 12 months), chronic lower respiratory tract disease, history of stroke/SE, history of bleeding-related hospitalisation, history of anaemia, use of cholesterol lowering drugs, use of antiplatelet drugs and use of NSAIDs during the last 12 months. * For risk of all-cause mortality, multivariate Cox proportional regression adjusting for the same variables used in competing risk regression was performed. NOAC, non-vitamin K antagonist oral anticoagulant; NSAIDs, non-steroidal antiinflammatory drugs; SE, systemic embolism.

2.11 (standard dose) and 2.33 (reduced dose) for dabigatran, 3.21 (standard dose) and 3.03 (reduced dose) for rivaroxaban, 3.08 (standard dose) and 3.77 (reduced dose) for apixaban and 2.69 for warfarin. The crude incidence rate of major bleeding (events per 100 patient years) was 1.78 (standard dose) and 2.24 (reduced dose) for users of dabigatran, 2.58 (standard dose) and 3.56 (reduced dose) for rivaroxaban, 2.22 (standard dose) and 3.04 (reduced dose) for apixaban and 3.02 for warfarin. The cumulative incidence functions for stroke/SE and major bleeding for each OAC are shown in figure 2.

\section{NOAC-warfarin comparisons}

Results of the comparisons between NOACs and warfarin for the main outcomes stroke/SE and major bleeding, as well as ischaemic stroke, intracranial haemorrhage, gastrointestinal bleeding, any bleeding and all-cause mortality are shown in figure 3 . We found similar risks of stroke/SE for both standard and reduced doses of all NOACs compared with warfarin. Both doses of apixaban were associated with lower risk of major bleeding compared with warfarin (standard dose SHR 0.74, 95\% CI 0.60 to 0.91 ; reduced dose SHR $0.78,95 \%$ CI 0.64 to 0.96 ), while use of both doses of dabigatran and rivaroxaban was associated with similar risks. For risk of all-cause mortality, no significant differences were found between standard dose of NOACs and warfarin, while reduced dose rivaroxaban (HR 1.42, 95\% CI 1.25 to 1.61 ) and reduced dose apixaban (HR 1.38, 95\% CI 1.22 to 1.56 ) were associated with significantly higher risk.

\section{NOAC-NOAC comparisons}

The results of NOAC-NOAC comparisons are shown in figure 4. No significant differences were found in risk of stroke/SE, except in the comparison between reduced dose of dabigatran and reduced dose of apixaban (SHR $0.77,95 \%$ CI 0.60 to 0.98 ). Standard dose of apixaban was associated with significantly lower risk of major bleeding compared with standard dose of rivaroxaban (SHR 0.76, 95\% CI 0.62 to 0.95 ). Further, reduced doses of apixaban and of dabigatran were associated with significantly lower risk of major bleeding compared with reduced doses of rivaroxaban (figure 4).

\section{Sensitivity analyses}

The results of the sensitivity analyses (table 2) were in line with the main analyses with respect to the main outcomes stroke/ $\mathrm{SE}$ and major bleeding. Regarding risk of all-cause death, the sensitivity analyses showed greater diversity in the results. Of particular interest is that in the 'intention-to-treat-analyses', the risk of all-cause death was lower or similar with reduced dose of NOACs compared with warfarin.

\section{DISCUSSION}

In this nationwide cohort study of elderly patients $\geq 75$ years with $\mathrm{AF}$, we investigated risk of thromboembolic and bleeding events associated with use of standard and reduced doses of NOACs compared with warfarin and NOACs compared with NOACs. Comparing NOACs with warfarin, we found comparable rates of stroke/SE for both standard and reduced dose NOACs and that both doses of apixaban were associated with significantly lower risks of major bleeding. In the NOAC-NOAC comparisons, reduced dose dabigatran was associated with a significantly lower risk of stroke/SE than reduced dose apixaban, while reduced dose dabigatran as well as both doses of apixaban were associated with lower risks of major bleeding compared with the corresponding doses of rivaroxaban. The median age of patients 
Table 2 Sensitivity analyses

\begin{tabular}{|c|c|c|c|c|c|}
\hline & & Sensitivity analyses & & & \\
\hline & Main analysis* & 90-day gap periodt & True OAC naive $\ddagger$ & $\begin{array}{l}\text { Standardised to 12-month } \\
\text { follow-up§ }\end{array}$ & Intention to treat analysis \\
\hline Standard dose $\mathrm{I}$ & ACs vs warfarin & & & & \\
\hline Stroke/SE & Subhazard ratio $(95 \% \mathrm{CI})$ & & & & \\
\hline Warfarin & Ref & Ref & Ref & Ref & Ref \\
\hline Dabigatran & 0.80 (0.57 to 1.13$)$ & 0.82 (0.58 to 1.15$)$ & 0.80 (0.56 to 1.14$)$ & 0.95 (0.62 to 1.43 ) & 0.81 (0.62 to 1.08 ) \\
\hline Rivaroxaban & 1.07 (0.89 to 1.30$)$ & 1.08 (0.89 to 1.30$)$ & 1.02 (0.84 to 1.25$)$ & 1.02 (0.80 to 1.30$)$ & 0.97 (0.83 to 1.14$)$ \\
\hline Apixaban & 0.95 (0.78 to 1.15$)$ & 0.95 (0.78 to 1.16$)$ & 0.92 (0.75 to 1.22 ) & 1.00 (0.79 to 1.28$)$ & 0.88 (0.74 to 1.04$)$ \\
\hline Major bleeding & Subhazard ratio $(95 \% \mathrm{Cl})$ & & & & \\
\hline Warfarin & Ref & Ref & Ref & Ref & Ref \\
\hline Dabigatran & 0.75 (0.52 to 1.08 ) & 0.73 (0.51 to 1.05$)$ & 0.77 (0.73 to 1.12 ) & 0.86 (0.53 to 1.39$)$ & 0.67 (0.50 to 0.87 ) \\
\hline Rivaroxaban & 0.96 (0.78 to 1.16$)$ & 0.95 (0.78 to 1.15$)$ & 0.90 (0.58 to 0.90$)$ & 0.88 (0.67 to 1.16$)$ & 0.84 (0.72 to 0.99 ) \\
\hline Apixaban & 0.74 (0.60 to 0.91 ) & 0.70 (0.57 to 0.87$)$ & 0.72 (0.58 to 0.90$)$ & 0.66 (0.50 to 0.86$)$ & 0.63 (0.53 to 0.75$)$ \\
\hline All-cause death & $\mathrm{HR}(95 \% \mathrm{Cl})$ & & & & \\
\hline Warfarin & Ref & Ref & Ref & Ref & Ref \\
\hline Dabigatran & 0.77 (0.57 to 1.05$)$ & 0.79 (0.54 to 0.91$)$ & 0.75 (0.55 to 1.02 ) & 0.76 (0.49 to 1.20$)$ & 0.66 (0.55 to 0.79$)$ \\
\hline Rivaroxaban & 1.12 (0.97 to 1.28$)$ & 0.90 (0.80 to 1.02 ) & 1.06 (0.91 to 1.23$)$ & 0.96 (0.78 to 1.18$)$ & 0.84 (0.77 to 0.92 ) \\
\hline Apixaban & 0.99 (0.85 to 1.15$)$ & 0.79 (0.70 to 0.91$)$ & 0.93 (0.79 to 1.08$)$ & 0.84 (0.69 to 1.03 ) & $0.72(0.65$ to 0.80$)$ \\
\hline Reduced dose $\mathrm{N}$ & Cs vs warfarin & & & & \\
\hline Stroke/SE & Subhazard ratio $(95 \% \mathrm{Cl})$ & & & & \\
\hline Warfarin & Ref & Ref & Ref & Ref & Ref \\
\hline Dabigatran & 0.87 (0.70 to 1.07 ) & 0.86 (0.70 to 1.06$)$ & 0.83 (0.66 to 1.03 ) & 0.91 (0.70 to 1.18 ) & 0.87 (0.74 to 1.03$)$ \\
\hline Rivaroxaban & 1.05 (0.85 to 1.30$)$ & 1.08 (0.87 to 1.33$)$ & 1.06 (0.85 to 1.32 ) & 1.19 (0.92 to 1.54$)$ & 1.06 (0.89 to 1.26$)$ \\
\hline Apixaban & 1.12 (0.92 to 1.38$)$ & 1.15 (0.94 to 1.41$)$ & 1.10 (0.89 to 1.35 ) & 1.15 (0.90 to 1.47$)$ & 1.00 (0.84 to 1.18$)$ \\
\hline Major bleeding & Subhazard ratio $(95 \% \mathrm{Cl})$ & & & & \\
\hline Warfarin & Ref & Ref & Ref & Ref & Ref \\
\hline Dabigatran & 0.85 (0.69 to 1.05$)$ & 0.83 (0.68 to 1.03 ) & 0.86 (0.68 to 1.07 ) & 0.86 (0.65 to 1.14$)$ & 0.87 (0.74 to 1.01 ) \\
\hline Rivaroxaban & 1.15 (0.95 to 1.40$)$ & 1.14 (0.94 to 1.38$)$ & 1.16 (0.94 to 1.42$)$ & 1.15 (0.89 to 1.48$)$ & 0.95 (0.81 to 1.12 ) \\
\hline Apixaban & 0.78 (0.64 to 0.96$)$ & 0.79 (0.65 to 0.96$)$ & 0.81 (0.66 to 1.00$)$ & 0.77 (0.60 to 0.99 ) & $0.67(0.57$ to 0.80$)$ \\
\hline All-cause death & $\mathrm{HR}(95 \% \mathrm{Cl})$ & & & & \\
\hline Warfarin & Ref & Ref & Ref & Ref & Ref \\
\hline Dabigatran & 1.11 (0.97 to 1.27 ) & 0.89 (0.79 to 1.00$)$ & 1.05 (0.91 to 1.21 ) & 1.08 (0.89 to 1.30$)$ & 0.89 (0.82 to 0.96$)$ \\
\hline Rivaroxaban & 1.42 (1.25 to 1.61$)$ & 1.14 (1.02 to 1.27 ) & 1.35 (1.18 to 1.54$)$ & 1.14 (0.95 to 1.36$)$ & 0.98 (0.91 to 1.07 ) \\
\hline Apixaban & 1.38 (1.22 to 1.56$)$ & 1.09 (0.99 to 1.22 ) & 1.34 (1.18 to 1.51$)$ & 1.24 (1.06 to 1.44$)$ & 0.96 (0.89 to 1.04 ) \\
\hline
\end{tabular}

* Multivariate competing risk regression, adjusted for NOAC dose, gender, age, year of inclusion into the study, chronic kidney disease, hypertension, diabetes, ischaemic heart disease, peripheral artery disease, heart failure, dementia, thyroid disorders, active cancer (cancer diagnosis last 12 months), chronic lower respiratory tract disease, history of stroke/SE, history of bleeding-related hospitalisation, history of anaemia, use of cholesterol lowering drugs, use of antiplatelet drugs and use of NSAIDs during the last 12 months, treating death as a competing risk. ${ }^{2}$

tAnalyses of the risk of stroke/SE and major bleeding among users of different OACs, allowing a longer gap period of 90 days between the calculated end of OAC supply and a new prescription dispensing before censoring.

$\ddagger$ Analyses of the risk of stroke/SE and major bleeding among users of different OACs, excluding patients with a dispensing of any anticoagulant from pharmacies during the last 5 years (12 months was used in the main analyses).

§Analyses of the risk of stroke/SE and major bleeding restricting follow-up time for all OACs to 12 months.

I|An 'intention-to-treat'-like analysis: investigating risk of stroke/SE and major bleeding without censoring by treatment switch or discontinuation of NOACs.

NOACs, non-vitamin K antagonist oral anticoagulants; NSAIDs, non-steroidal anti-inflammatory drugs; OACs, oral anticoagulants; SE, systemic embolism.

included was 82 years and the mean $\mathrm{CHA}_{2} \mathrm{DS}_{2}$-VASc score was 4.5 , implying that this was a truly high-risk population.

To our knowledge, this is one of the first studies of an allcomers nationwide cohort of patients with $\mathrm{AF} \geq 75$ years, investigating a less selected group of elderly patients than most previous observational studies. ${ }^{12} 13 \quad 2324$ Using high-quality nationwide registries with almost complete coverage ${ }^{14}$ reduces selection bias and eliminates loss-to-follow-up; important limitations for studies based on insurance claims databases (eligibility for insurance required) ${ }^{24}$ or prospective studies (healthy volunteer effect). ${ }^{13}$ Also, using administrative health registries reduces information bias as all diagnoses are coded according to the ICD-10 system.
Our findings were generally in line with subgroup analyses ${ }^{8-11}$ of the pivotal RCTs. ${ }^{4-6}$ From the RE-LY trial, ${ }^{4}$ subgroup analyses of the $7258(40 \%)$ patients $\geq 75$ years showed that the reduced risk of stroke/SE associated with dabigatran was maintained in the elderly population. ${ }^{11}$ Subgroup analyses of the 6229 (44\%) patients $\geq 75$ years included in the ROCKET-AF trial ${ }^{6}$ also showed a consistency in the effects of rivaroxaban versus warfarin regarding risk of stroke/SE across age groups, but a higher risk of major or clinically relevant non-major bleeding in patients $>75$ years. ${ }^{8}$ From the ARISTOTLE trial, ${ }^{5}$ subgroup analyses of the $5678(31 \%)$ patients included $\geq 75$ years showed that the benefits of apixaban in reducing risk of stroke/SE as well as major bleeding were maintained across all age groups. ${ }^{9}$ 
There are also some previous observational studies comparing NOACs versus warfarin in the elderly, with findings in line with our results. $^{12132324}$

In a recent meta-analysis including 22 studies enrolling over 440000 patients $\geq 75$ years, indirect comparisons between NOACs (Bucher method) showed no significant differences between NOACs for risk of stroke/SE, but significant differences in risk of major bleeding; apixaban was associated with significantly lower risk of major bleeding compared with both dabigatran and rivaroxaban, while there was no significant difference between dabigatran and rivaroxaban. ${ }^{25}$ Importantly, methods of indirect comparisons could systematically overestimate or underestimate treatment effect, warranting cautious interpretation. ${ }^{26}$

Regarding all-cause death, we found similar risks for standard doses of all three NOACs compared with warfarin, while reduced doses of rivaroxaban and apixaban were associated with a significantly higher risk of all-cause mortality. This was unexpected, as the RCTs on NOACs versus warfarin showed similar or favourable risks of all-cause mortality. We believe this discrepancy is due to unmeasured confounders. First, we did not have information about body mass index, estimated glomerular filtration rate and frailty-factors important in choice of anticoagulant dose and also affecting risk of death. Second, lack of knowledge of these factors made it impossible to assess appropriateness of dosage. A recent study from the Global Anticoagulant Registry in the FIELD-AF (GARFIELD-AF) investigated degree of recommended and non-recommended dosing of NOACs among 10426 patients with AF and found that 23.2\% were underdosed and 3.8\% were overdosed. ${ }^{27}$ Prescription of non-recommended doses was associated with a higher risk of allcause mortality (HR 1.24, 95\% CI 1.04 to 1.48). Patient characteristics leading clinicians to choose a non-recommended low dose are difficult to identify and adjust for, but influential for risk of all-cause mortality. Third, the sensitivity analyses showed consistency in all comparisons for stroke/SE and major bleeding, but great diversity for the risk of all-cause mortality, particularly when comparing reduced doses of NOACs with warfarin. This supports a stronger influence of residual confounding for this outcome, leading us to de-emphasise our findings.

A net clinical benefit of oral anticoagulation in elderly patients with AF has been shown in several studies, ${ }^{2} 28$ but still many clinicians withhold anticoagulants due to fear of bleeding complications. $^{30}$ This study might increase physician confidence in prescribing OACs to this vulnerable high-risk group of patients.

\section{Strengths and limitations}

With the active-comparator design, we tried to reduce confounding by indication. However, unknown/unmeasurable confounders are inevitably present in observational studies, leading to residual confounding. Outcomes were not adjudicated, thus miscoding and under-reporting will be present, but likely equally for all NOACs. Information about the reason for dose reduction of NOACs was lacking, and some patients may have received non-recommended reduced doses. We therefore analysed standard and reduced dose NOACs separately. Furthermore, the criteria warranting dose reduction vary between NOACs, complicating comparisons. Perhaps most notably, in Europe the reduced dose dabigatran is recommended for all patients $\geq 80$ years. $^{3}$ Accordingly, the reduced dose may be viewed as 'standard' for elderly patients using dabigatran. No subgroup analyses with respect to age were performed due to concern with statistical power. We studied use of OACs according to prescriptions dispensed, not drugs actually taken.
Finally, this study describes associations rather than drawing causal inferences.

\section{CONCLUSION}

In this real-world study of patients $\geq 75$ years initiating oral anticoagulation for AF, standard and reduced dose NOACs were associated with similar risks of stroke/SE as warfarin and lower or similar risks of bleeding. The NOACs seem to be a safe option also in elderly patients.

\section{Key messages}

What is already known on this subject?

- Non-vitamin K antagonist oral anticoagulants (NOACs) are firmly established as the preferred class of drugs for stroke prophylaxis in atrial fibrillation (AF). No randomised controlled trials (RCTs) have specifically investigated the efficacy and safety of NOACs compared with warfarin or NOACs compared with NOACs, among elderly patients with AF.

\section{What might this study add?}

- This real-world study adds insight into the comparative effectiveness and safety of NOACs in the elderly population with AF compared with warfarin but also when compared with each other. It supports the findings from subgroup analyses of the pivotal RCTs comparing NOAC versus warfarin, that NOACs are an effective and safe option also for elderly patients with AF with their higher stroke and bleeding risk.

How might this impact on clinical practice?

- The results from this study could increase physician confidence in prescribing oral anticoagulants for elderly patients. It could also serve as a hypothesis generator for RCTs comparing NOAC versus NOAC.

Contributors $0-C W R$ is the first author and performed all analyses, as well as drafting the manuscript. O-CWR, SH, WG and CJ were closely involved in the planning, conduct and reporting of this article. FS was involved in all statistical analyses, as well as reporting. SH was responsible for the overall content of the article.

Funding This work was funded by the South-Eastern Norway Regional Health Authority (no grant number).

Competing interests 0 -CWR reports personal fees from Novartis, Boehringer Ingelheim and Merck, outside the submitted work. CJ reports personal fees from BMS/Pfizer and Bayer, outside the submitted work. WG reports grants and personal fees from Bayer, MSD, Novartis, Amgen and Sanofi, outside the submitted work. SH reports speaker fees from BMS/Pfizer, Bayer and Boehringer-Ingelheim, outside the submitted work.

Patient consent for publication Not required.

Ethics approval Registration of data into the NPR and the NorPD is mandatory in Norway and legally exempt from obtainment of patient consent. This study was approved by the Regional Ethics Committee (Ref. No. 2017/410/REK North).

Provenance and peer review Not commissioned; externally peer reviewed.

Data availability statement No data are available. The authors are not permitted to share data to other researchers without due application process.

Supplemental material This content has been supplied by the author(s). It has not been vetted by BMJ Publishing Group Limited (BMJ) and may not have been peer-reviewed. Any opinions or recommendations discussed are solely those of the author(s) and are not endorsed by BMJ. BMJ disclaims all liability and responsibility arising from any reliance placed on the content. Where the content includes any translated material, BMJ does not warrant the accuracy and reliability of the translations (including but not limited to local regulations, clinical guidelines, terminology, drug names and drug dosages), and 
is not responsible for any error and/or omissions arising from translation and adaptation or otherwise.

Open access This is an open access article distributed in accordance with the Creative Commons Attribution Non Commercial (CC BY-NC 4.0) license, which permits others to distribute, remix, adapt, build upon this work non-commercially, and license their derivative works on different terms, provided the original work is properly cited, appropriate credit is given, any changes made indicated, and the use is non-commercial. See: http://creativecommons.org/licenses/by-nc/4.0/.

ORCID iD

Ole-Christian Walter Rutherford http://orcid.org/0000-0001-5721-9917

\section{REFERENCES}

1 Mitrousi K, Lip GYH, Apostolakis S. Age as a risk factor for stroke in atrial fibrillation patients: implications in thromboprophylaxis in the era of novel oral anticoagulants. $J$ Atr Fibrillation 2013;6:783.

2 Karamichalakis N, Georgopoulos S, Vlachos K, et al. Efficacy and safety of novel anticoagulants in the elderly. J Geriatr Cardiol 2016;13:718-23.

3 Hindricks G, Potpara T, Dagres N, et al. 2020 ESC guidelines for the diagnosis and management of atrial fibrillation developed in collaboration with the European association for Cardio-Thoracic surgery (EACTS). Eur Heart J 2021;42:373-498.

4 Connolly SJ, Ezekowitz MD, Yusuf S, et al. Dabigatran versus warfarin in patients with atrial fibrillation. N Eng/ J Med 2009;361:1139-51.

5 Granger $\mathrm{CB}$, Alexander JH, McMurray JJV, et al. Apixaban versus warfarin in patients with atrial fibrillation. N Engl J Med 2011;365:981-92.

6 Patel MR, Mahaffey KW, Garg J, et al. Rivaroxaban versus warfarin in nonvalvular atrial fibrillation. N Eng/ J Med 2011;365:883-91.

7 Van Ganse E, Danchin N, Mahé I, et al. Comparative safety and effectiveness of oral anticoagulants in nonvalvular atrial fibrillation: the Naxos study. Stroke 2020;51:2066-75.

8 Halperin JL, Hankey GJ, Wojdyla DM, et al. Efficacy and safety of rivaroxaban compared with warfarin among elderly patients with nonvalvular atrial fibrillation in the rivaroxaban once daily, oral, direct factor Xa inhibition compared with vitamin $\mathrm{K}$ antagonism for prevention of stroke and embolism trial in atrial fibrillation (rocket AF). Circulation 2014:130:138-46.

9 Halvorsen S, Atar D, Yang H, et al. Efficacy and safety of apixaban compared with warfarin according to age for stroke prevention in atrial fibrillation: observations from the ARISTOTLE trial. Eur Heart J 2014;35:1864-72.

10 Kato ET, Giugliano RP, Ruff CT, et al. Efficacy and safety of edoxaban in elderly patients with atrial fibrillation in the engage AF-TIMI 48 trial. J Am Heart Assoc 2016:5:e003432.

11 Lauw MN, Eikelboom JW, Coppens M, et al. Effects of dabigatran according to age in atrial fibrillation. Heart 2017;103:1015-23.

12 Chao T-F, Chiang C-E, Liao J-N, et al. Comparing the effectiveness and safety of Nonvitamin $\mathrm{K}$ antagonist oral anticoagulants and warfarin in elderly Asian patients with atrial fibrillation: a nationwide cohort study. Chest 2020;157:1266-77.
13 Patti G, Pecen L, Lucerna M, et al. Net clinical benefit of non-vitamin K antagonist vs vitamin $\mathrm{K}$ antagonist anticoagulants in elderly patients with atrial fibrillation. Am J Med 2019;132:749-57.

14 Bakken IJ, Ariansen AMS, Knudsen GP, et al. The Norwegian Patient Registry and the Norwegian Registry for Primary Health Care: research potential of two nationwide health-care registries. Scand J Public Health 2020;48:49-55.

15 NOMESCO Classification of Surgical Procedures (NCSP), version 1.16 [Internet], 2011 Available: http://norden.diva-portal.org/smash/record.jsf?pid=diva2\%3A968721\& dswid=-7662 [Accessed 02 Feb 2021].

16 Wettermark B, Zoëga H, Furu K, et al. The Nordic prescription databases as a resource for pharmacoepidemiological research--a literature review. Pharmacoepidemiol Drug Saf 2013;22:691-9.

17 Yoshida K, Solomon DH, Kim SC. Active-comparator design and new-user design in observational studies. Nat Rev Rheumatol 2015;11:437-41.

18 Halvorsen S, Ghanima W, Fride Tvete I, et al. A nationwide registry study to compare bleeding rates in patients with atrial fibrillation being prescribed oral anticoagulants. Eur Heart J Cardiovasc Pharmacother 2017:3:28-36.

19 Lesko CR, Lau B. Bias due to confounders for the Exposure-Competing risk relationship. Epidemiology 2017:28:20-7.

20 Fine JP, Gray RJ. A proportional hazards model for the Subdistribution of a competing risk. J Am Stat Assoc 1999:94:496-509.

21 Wolbers M, Koller MT, Stel VS, et al. Competing risks analyses: objectives and approaches. Eur Heart J 2014;35:2936-41.

22 Lin DY, Wei LJ. The robust inference for the COX proportional hazards model. J Am Stat Assoc 1989;84:1074-8

23 Forslund T, Wettermark B, Andersen M, et al. Stroke and bleeding with non-vitamin $\mathrm{K}$ antagonist oral anticoagulant or warfarin treatment in patients with non-valvular atrial fibrillation: a population-based cohort study. Europace 2018;20:420-8.

24 Lip GYH, Keshishian A, Li X, et al. Effectiveness and safety of oral anticoagulants among nonvalvular atrial fibrillation patients. Stroke 2018;49:2933-44.

25 Silverio A, Di Maio M, Prota C, et al. Safety and efficacy of non-vitamin K antagonist oral anticoagulants in elderly patients with atrial fibrillation: systematic review and meta-analysis of 22 studies and 440281 patients. Eur Heart J Cardiovasc Pharmacother 2021;7:f20-9

26 Mills EJ, Ghement I, O'Regan C, et al. Estimating the power of indirect comparisons: a simulation study. PLoS One 2011;6:e16237.

27 Camm AJ, Cools F, Virdone S, et al. Mortality in patients with atrial fibrillation receiving Nonrecommended doses of direct oral anticoagulants. J Am Coll Cardiol 2020;76:1425-36.

$28 \mathrm{Ng} \mathrm{KH}$, Shestakovska O, Connolly SJ, et al. Efficacy and safety of apixaban compared with aspirin in the elderly: a subgroup analysis from the AVERROES trial. Age Ageing 2016;45:77-83.

29 Patti G, Lucerna M, Pecen L, et al. Thromboembolic Risk, Bleeding Outcomes and Effect of Different Antithrombotic Strategies in Very Elderly Patients With Atrial Fibrillation: $A$ Sub-Analysis From the PREFER in AF (PREvention of Thromboembolic Events-European Registry in Atrial Fibrillation). J Am Heart Assoc 2017:6:e005657.

30 Chao T-F, Liu C-J, Lin Y-J, et al. Oral anticoagulation in very elderly patients with atrial fibrillation: a nationwide cohort study. Circulation 2018:138:37-47. 